\title{
On the distribution of maximal percentages in pólya's urn
}

DOI:

$10.1017 /$ S0269964816000358

\section{Document Version}

Accepted author manuscript

Link to publication record in Manchester Research Explorer

\section{Citation for published version (APA):}

Nadarajah, S. (2017). On the distribution of maximal percentages in pólya's urn. Probability in the Engineering and Informational Sciences, 31(3), 357-365. https://doi.org/10.1017/S0269964816000358

\section{Published in:}

Probability in the Engineering and Informational Sciences

\section{Citing this paper}

Please note that where the full-text provided on Manchester Research Explorer is the Author Accepted Manuscript or Proof version this may differ from the final Published version. If citing, it is advised that you check and use the publisher's definitive version.

\section{General rights}

Copyright and moral rights for the publications made accessible in the Research Explorer are retained by the authors and/or other copyright owners and it is a condition of accessing publications that users recognise and abide by the legal requirements associated with these rights.

\section{Takedown policy}

If you believe that this document breaches copyright please refer to the University of Manchester's Takedown Procedures [http://man.ac.uk/04Y6Bo] or contact uml.scholarlycommunications@manchester.ac.uk providing relevant details, so we can investigate your claim.

\section{OPEN ACCESS}




\title{
On the distribution of maximal percentages in Pólya's urn
}

by

Saralees Nadarajah

School of Mathematics, University of Manchester, Manchester M13 9PL, UK

\begin{abstract}
Schulte-Geers and Stadje [Journal of Applied Probability, 52, 2015, 180-190] gave several closed form expressions for the exact distribution of the all-time maximal percentage in Pólya's urn model. But all these expressions corresponded to an integer parameter taking the value 1. Here, we derive much more general closed form expressions applicable for all possible values of the integer parameter. We also illustrate their computational efficiency.
\end{abstract}

Keywords: Closed form expression; Elementary expression; Hypergeometric function

\section{Introduction}

Suppose that an urn contains $r \geq 1$ red balls and $b \geq 1$ black balls. In each draw, a ball is selected at random and that ball and $d \geq 1$ balls of the same color are put into the urn. This is known as the scheme due to Eggenberger-Pólya (1923). There are extensive theoretical developments on this scheme, see Mahmoud (2009) for an excellent review. This note concerns the latest contribution due to Schulte-Geers and Stadje (2015).

Let $R_{n}, n \geq 1$ denote the number of red balls in the urn after the $n$th draw. Let $R_{0}=r$. Let $Z_{n}=R_{n} /(n d+r+b)$. Then

$$
S_{r, b}=\sup _{n \geq 0} Z_{n}
$$

is the supremum of all successive percentages of red balls during the entire drawing process. SchulteGeers and Stadje (2015) derived several expressions for

$$
P\left(S_{r, b}>\frac{t-1}{t}\right)=P(d, r, b, t) \text { say. }
$$

The expressions were in closed form when: $d=1, r=1, b=t-1$ (top of page 181 and Proposition 5.4 ); $d=1, r=1, b=1$ (top of page 181 and Proposition 5.2); $d=1, r=a, b=a, t=2$ (top of page 190). The expressions were presented as infinite series when: $r \geq 1, b \geq 1, t \geq 2$ (bottom of page 180 and Proposition 5.1); $d=1, r=t-1, b=1$ (middle of page 181 and Proposition 5.3).

The aim of this note is to derive closed form expressions for $P(d, r, b, t)$ for a much wider range of values $d, r, b$ and $t$. Infinite series are usually not regarded as closed form expressions. However, special functions (especially those for which in-built routines are widely available) defined in terms of infinite series can be referred to as closed form expressions. We also illustrate computational efficiency of the derived closed form expressions over the infinite series representations in SchulteGeers and Stadje (2015) for a wide range of values of $d, r, b$ and $t$. 
The technique used expresses $P(d, r, b, t)$ in the general case in terms of a well known and well established hypergeometric function, see Section 2. Some details of this hypergeometric function can be found in Prudnikov et al. (1986) and Gradshteyn and Ryzhik (2014). There are many other books giving detailed properties of the hypergeometric function. These properties can be used to derive elementary and other closed form expressions for $P(d, r, b, t)$. This is illustrated in Sections 3, 4, 5 and 6, using properties in Sections 2.20 to 2.22 of Prudnikov et al. (1986, volume 3).

We must emphasize that the elementary / closed form expressions for $P(d, r, b, t)$ given in Sections 3 to 6 are only a small selection. The number of elementary / closed form expressions for $P(d, r, b, t)$ derivable using the result of Section 2 is possibly uncountable. The readers may want to derive other elementary / closed form expressions not listed in Sections 3 to 6.

\section{Closed for expression for $P(d, r, b, t)$ in the general case}

By Proposition 5.1 in Schulte-Geers and Stadje (2015),

$$
P(d, r, b, t)=\sum_{n=0}^{\infty} \frac{a+1}{n(t-1)+a+1}\left(\begin{array}{c}
n t+a \\
n
\end{array}\right) \frac{B(n(t-1)+a+1+r / d, n+b / d)}{B(r / d, b / d)},
$$

where $a=[m / d], m=b(t-1)-r \geq 0$ and

$$
B(a, b)=\int_{0}^{1} t^{a-1}(1-t)^{b-1} d t
$$

denotes the beta function.

Theorem 2.1 expresses (1) in terms of the hypergeometric function

$$
{ }_{p} F_{q}\left(a_{1}, a_{2}, \ldots, a_{p} ; b_{1}, b_{2}, \ldots, b_{q} ; x\right)=\sum_{k=0}^{\infty} \frac{\left(a_{1}\right)_{k}\left(a_{2}\right)_{k} \cdots\left(a_{p}\right)_{k}}{\left(b_{1}\right)_{k}\left(b_{2}\right)_{k} \cdots\left(b_{q}\right)_{k}} \frac{x^{k}}{k !},
$$

where $(a)_{k}=\Gamma(k+a) / \Gamma(a)=a(a+1) \cdots(a+k-1)$ denotes the ascending factorial and

$$
\Gamma(a)=\int_{0}^{\infty} t^{a-1} \exp (-t) d t
$$

denotes the gamma function.

In-built routines for computing ${ }_{p} F_{q}\left(a_{1}, a_{2}, \ldots, a_{p} ; b_{1}, b_{2}, \ldots, b_{q} ; x\right)$ are widely available in packages like Maple, Matlab and Mathematica. For example, $P F Q\left[\left\{a_{1}, a_{2}, \ldots, a_{p}\right\},\left\{b_{1}, b_{2}, \ldots, b_{q}\right\}, x\right]$ in Mathematica computes ${ }_{p} F_{q}\left(a_{1}, a_{2}, \ldots, a_{p} ; b_{1}, b_{2}, \ldots, b_{q} ; x\right)$. Mathematica like other algebraic manipulation packages allows for arbitrary precision, so the accuracy of computation is not an issue.

Theorem 2.1 For $d \geq 1, r \geq 1, b \geq 1$ and $t \geq 1$,

$$
\begin{aligned}
P(d, r, b, t)=C(d, r, b, t){ }_{2 t} F_{2 t-1}\left(\frac{a+1}{t}, \frac{a+2}{t}, \ldots, \frac{a+t}{t},\right. \\
\quad \frac{a+1+\frac{r}{d}, \frac{a+2+\frac{r}{d}}{t-1}, \ldots, \frac{a+t-1+\frac{r}{d}}{t-1}, \frac{b}{d} ;}{} \\
\left.\frac{a+1+\frac{r+b}{d}}{t}, \frac{a+2+\frac{r+b}{d}}{t}, \ldots, \frac{a+t+\frac{r+b}{d}}{t}, \frac{a+2}{t-1}, \frac{a+3}{t-1}, \ldots, \frac{a+t}{t-1} ; 1\right),
\end{aligned}
$$


where

$$
C(d, r, b, t)=\frac{(a+1) \Gamma\left(\frac{r+b}{d}\right)(t-1)^{\frac{r}{d}-1}}{\Gamma\left(\frac{r}{d}\right) t^{\frac{r+b}{d}}} \prod_{k=0}^{t-1} \frac{\Gamma\left(\frac{a+1+k}{t}\right)}{\Gamma\left(\frac{a+1+k+\frac{r+b}{d}}{t}\right)} \prod_{k=0}^{t-2} \frac{\Gamma\left(\frac{a+1+k+\frac{r}{d}}{t-1}\right)}{\Gamma\left(\frac{a+2+k}{t-1}\right)} .
$$

Proof: Using the fact $B(a, b)=\Gamma(a) \Gamma(b) / \Gamma(a+b)$, we can rewrite (1) as

$$
P(d, r, b, t)=\frac{a+1}{B(r / d, b / d)} \sum_{n=0}^{\infty} \frac{\Gamma(n t+a+1)}{\Gamma\left(n t+a+1+\frac{r+b}{d}\right)} \frac{\Gamma\left(n(t-1)+a+1+\frac{r}{d}\right)}{\Gamma(n(t-1)+a+2)} \frac{\Gamma\left(n+\frac{b}{d}\right)}{n !} .
$$

Using the fact

$$
\Gamma(t z+b)=t^{t z+b-\frac{1}{2}}(2 \pi)^{\frac{1-t}{2}} \prod_{k=0}^{t-1} \Gamma\left(z+\frac{b+k}{t}\right)
$$

we can write

$$
\begin{aligned}
\frac{\Gamma(n t+a+1)}{\Gamma\left(n t+a+1+\frac{r+b}{d}\right)} & =t^{-\frac{r+b}{d}} \prod_{k=0}^{t-1} \frac{\Gamma\left(n+\frac{a+1+k}{t}\right)}{\Gamma\left(n+\frac{a+1+k+\frac{r+b}{d}}{t}\right)} \\
& =t^{-\frac{r+b}{d}} \prod_{k=0}^{t-1} \frac{\Gamma\left(\frac{a+1+k}{t}\right)}{\Gamma\left(\frac{a+1+k+\frac{r+b}{d}}{t}\right)} \prod_{k=0}^{t-1} \frac{\left(\frac{a+1+k}{t}\right)_{n}}{\left(\frac{a+1+k+\frac{r+b}{d}}{t}\right)_{n}} .
\end{aligned}
$$

Similarly, we can write

$$
\frac{\Gamma\left(n(t-1)+a+1+\frac{r}{d}\right)}{\Gamma(n(t-1)+a+2)}=(t-1)^{\frac{r}{d}-1} \prod_{k=0}^{t-2} \frac{\Gamma\left(\frac{a+1+k+\frac{r}{d}}{t-1}\right)}{\Gamma\left(\frac{a+2+k}{t-1}\right)} \prod_{k=0}^{t-2} \frac{\left(\frac{a+1+k+\frac{r}{d}}{t-1}\right)_{n}}{\left(\frac{a+2+k}{t-1}\right)_{n}} .
$$

The result follows by combining (4)-(6) and using the definition of the hypergeometric function.

A hypergeometric form for $P(d, r, b, 2)$ appears to have been noted before by Antal et al. (2010).

Figure 1 illustrates the computational efficiency of (2) versus (1). The central processing unit times taken for one hundred computations of (1) and (2) are plotted versus:

- $d=1,2, \ldots, 100$ when $r=1, b=1$ and $t=2$ (top left figure);

- $r=1,2, \ldots, 100$ when $d=1, b=100$ and $t=2$ (top right figure);

- $b=1,2, \ldots, 100$ when $d=1, r=1$ and $t=2$ (bottom left figure);

- $t=2,3, \ldots, 101$ when $d=1, r=1$ and $b=1$ (bottom right figure).

We can see that the central processing unit times for (2) are about two times smaller across all values of $(d, r, b, t)$. The central processing unit times do not appear to change much with respect to $d, r$ or $b$. However, they do appear to increase with increasing $t$. All of the computations were performed using Mathematica.

We also performed similar studies for the expressions given in Sections 3 to 6 , the details of which are not presented here to avoid repetitive discussion. The central processing unit times taken for one hundred computations of these expressions appeared smaller than the times taken for one hundred computations of (2). That is, the expressions given in Sections 3 to 6 appeared computationally even more efficient than (1). 
Figure 1: Central processing unit times taken for one hundred computations of (1) (solid line) and (2) (broken line).

\section{Case $t=2$}

In this case, (2) can be reduced to the elementary form

$$
\begin{aligned}
& P(d, r, b, t)=C(d, r, b)(-1)^{-\frac{a+3}{2}-\frac{r+b}{2 d}} \Gamma\left(\frac{a+2}{2}+\frac{r+b}{2 d}\right) \Gamma(a+2) \sqrt{\Gamma\left(\frac{1-a}{2}\right)} \sqrt{\Gamma\left(-\frac{a}{2}\right)} \\
& \cdot \sqrt{\Gamma\left(-a-\frac{r}{d}\right)} \sqrt{\Gamma\left(1-\frac{b}{d}\right)} \sqrt{\Gamma\left(1-\frac{r+b}{2 d}\right)} \sqrt{\Gamma\left(\frac{1}{2}-\frac{r+b}{2 d}\right)} \\
& \cdot \sqrt{\Gamma\left(\frac{a+1}{2}+\frac{r-b}{2 d}\right)} \sqrt{\Gamma\left(-\frac{a+1}{2}+\frac{b-r}{2 d}\right)} \\
& \left\{\begin{array}{ccc}
\frac{a+3}{4}-\frac{b}{2 d} & \frac{2 b-r}{4 d}-\frac{3}{4} & \frac{a}{4}-\frac{r+b}{4 d} \\
-\frac{a}{4}-\frac{1}{2}-\frac{r}{2 d} & \frac{r-b}{4 d}-\frac{1}{2} & \frac{a-1}{4}+\frac{r+b}{4 d}
\end{array}\right\} \\
& /\left[\Gamma\left(\frac{1-a}{2}-\frac{r+b}{2 d}\right) \sqrt{\Gamma\left(\frac{a+3}{2}\right)} \sqrt{\Gamma\left(\frac{r+b}{2 d}\right)} \sqrt{\Gamma\left(\frac{b-r}{2 d}-\frac{a}{2}\right)}\right. \\
& \cdot \sqrt{\Gamma\left(\frac{a+2}{2}+\frac{r-b}{2 d}\right)} \\
& \left.\cdot \sqrt{\Gamma\left(\frac{1}{2}+\frac{r+b}{2 d}\right)} \sqrt{\Gamma\left(\frac{a+2}{2}\right)} \sqrt{\Gamma\left(1-\frac{r}{d}\right)} \sqrt{\Gamma\left(a+2-\frac{b}{d}\right)}\right],
\end{aligned}
$$

where

$$
C(d, r, b)=\frac{(a+1) \Gamma\left(\frac{r+b}{d}\right)}{\Gamma\left(\frac{r}{d}\right) 2^{\frac{r+b}{d}}} \frac{\Gamma\left(\frac{a+1}{2}\right)}{\Gamma\left(\frac{a+1+\frac{r+b}{d}}{2}\right)} \frac{\Gamma\left(\frac{a+2}{2}\right)}{\Gamma\left(\frac{a+2+\frac{r+b}{d}}{2}\right)} \frac{\Gamma\left(a+1+\frac{r}{d}\right)}{\Gamma(a+2)}
$$

and $\left\{\begin{array}{lll}j_{1} & j_{2} & j_{3} \\ j_{4} & j_{5} & j_{6}\end{array}\right\}$ is the Racah six $j$ symbol defined by

$$
\begin{aligned}
& {\left[\sqrt{\left(j_{1}+j_{2}-j_{3}\right) !} \sqrt{\left(j_{1}-j_{2}+j_{3}\right) !} \sqrt{\left(-j_{1}+j_{2}+j_{3}\right) !} \sqrt{\left(j_{3}+j_{4}-j_{5}\right) !}\right.} \\
& \cdot \sqrt{\left(j_{3}-j_{4}+j_{5}\right) !} \sqrt{\left(-j_{3}+j_{4}+j_{5}\right) !} \sqrt{\left(j_{1}+j_{5}-j_{6}\right) !} \sqrt{\left(j_{1}-j_{5}+j_{6}\right) !} \\
& \left.\cdot \sqrt{\left(-j_{1}+j_{5}+j_{6}\right) !} \sqrt{\left(j_{2}+j_{4}-j_{6}\right) !} \sqrt{\left(j_{2}-j_{4}+j_{6}\right) !} \sqrt{\left(-j_{2}+j_{4}+j_{6}\right) !}\right] \\
& \quad \cdot\left[\sqrt{\left(j_{1}+j_{2}+j_{3}+1\right) !} \sqrt{\left(j_{3}+j_{4}+j_{5}+1\right) !} \sqrt{\left(j_{1}+j_{5}+j_{6}+1\right) !} \sqrt{\left(j_{2}+j_{4}+j_{6}+1\right) !}\right] \\
& \quad \cdot \sum_{k=p}^{q} \frac{(-1)^{k}(k+1) !\left[\left(j_{1}+j_{2}+j_{4}+j_{5}-k\right) !\left(j_{1}+j_{3}+j_{4}+j_{6}-k\right) !\left(j_{2}+j_{3}+j_{5}+j_{6}-k\right) !\right.}{\left(k-j_{1}-j_{2}-j_{3}\right) !\left(k-j_{3}-j_{4}-j_{5}\right) !\left(k-j_{1}-j_{5}-j_{6}\right) !\left(k-j_{2}-j_{4}-j_{6}\right) !}
\end{aligned}
$$

where $p=\max \left(j_{1}+j_{2}+j_{3}, j_{3}+j_{4}+j_{5}, j_{1}+j_{5}+j_{6}, j_{2}+j_{4}+j_{6}\right)$ and $q=\min \left(j_{1}+j_{2}+j_{4}+j_{5}\right.$, $\left.j_{1}+j_{3}+j_{4}+j_{6}, j_{2}+j_{3}+j_{5}+j_{6}\right)$. 


\section{Case $r / d=1$}

In this case, (2) can be reduced to

$$
\begin{aligned}
P(d, r, b, t)= & C(d, b, t)_{2 t} F_{2 t-1}\left(\frac{a+1}{t}, \frac{a+2}{t}, \ldots, \frac{a+t}{t}, \frac{a+2}{t-1}, \frac{a+3}{t-1}, \ldots, \frac{a+t}{t-1}, \frac{b}{d} ;\right. \\
& \left.\frac{a+2+\frac{b}{d}}{t}, \frac{a+3+\frac{b}{d}}{t}, \ldots, \frac{a+t+1+\frac{b}{d}}{t}, \frac{a+2}{t-1}, \frac{a+3}{t-1}, \ldots, \frac{a+t}{t-1} ; 1\right) \\
= & C(d, b, t)_{t+1} F_{t}\left(\frac{a+1}{t}, \frac{a+2}{t}, \ldots, \frac{a+t}{t}, \frac{b}{d} ;\right. \\
& \left.\frac{a+2+\frac{b}{d}}{t}, \frac{a+3+\frac{b}{d}}{t}, \ldots, \frac{a+t+1+\frac{b}{d}}{t} ; 1\right)
\end{aligned}
$$

where

$$
C(d, b, t)=\frac{(a+1) \Gamma\left(1+\frac{b}{d}\right)}{t^{1+\frac{b}{d}}} \prod_{k=0}^{t-1} \frac{\Gamma\left(\frac{a+1+k}{t}\right)}{\Gamma\left(\frac{a+2+k+\frac{b}{d}}{t}\right)} .
$$

Note that ${ }_{t+1} F_{t}$ is a simpler hypergeometric function than ${ }_{2 t} F_{2 t-1}$. (7) can be reduced to elementary functions for at least $t=2,3$. If $t=2$ then (7) can be reduced to

$$
\begin{gathered}
P(d, r, b, t)=C(d, b) \quad \sqrt{\Gamma\left(\frac{1-a}{2}\right)} \sqrt{\Gamma\left(-\frac{a}{2}\right)} \sqrt{\Gamma\left(1-\frac{b}{d}\right)} \Gamma\left(\frac{a+2}{2}+\frac{b}{2 d}\right) \Gamma\left(\frac{3+a}{2}+\frac{b}{2 d}\right) \\
\cdot\left\langle\frac{b}{2 d}-\frac{a+1}{4} \frac{1}{2}-\frac{b}{2 d} \frac{a+1}{4}+\frac{b}{4 d}-\frac{3 b}{4 d}\right. \\
\left|\frac{b}{2 d}-\frac{a+1}{4} \frac{1}{2}-\frac{b}{2 d} \frac{a-1}{4} \frac{a+1}{4}-\frac{b}{2 d}\right\rangle \\
/\left[\sqrt{\frac{a+1}{2} \Gamma\left(\frac{1}{2}+\frac{b}{2 d}\right) \sqrt{\Gamma\left(\frac{b}{2 d}\right)} \sqrt{\Gamma\left(\frac{a+2}{2}-\frac{b}{2 d}\right)}}\right. \\
\cdot \sqrt{\Gamma\left(1+\frac{b}{2 d}\right)} \sqrt{\left.\Gamma\left(\frac{a+3}{2}-\frac{b}{2 d}\right)\right]},
\end{gathered}
$$

where

$$
C(d, b)=\frac{(a+1) \Gamma\left(1+\frac{b}{d}\right)}{2^{1+\frac{b}{d}}} \frac{\Gamma\left(\frac{a+1}{2}\right)}{\Gamma\left(\frac{a+2+\frac{b}{d}}{2}\right)} \frac{\Gamma\left(\frac{a+2}{2}\right)}{\Gamma\left(\frac{a+3+\frac{b}{d}}{2}\right)}
$$

and $\left\langle j_{1} j_{2} m_{1} m_{2} \mid j_{1} j_{2} j m\right\rangle$ is the Clebsch-Gordan coefficient defined by

$$
\begin{aligned}
& \frac{\delta_{m, m_{1}+m_{2}}}{\sqrt{\left(j+j_{1}+j_{2}+1\right) !}} \sqrt{\left(j_{1}+j_{2}-j\right) !} \sqrt{\left(j+j_{1}-j_{2}\right) !} \sqrt{\left(j-j_{1}+j_{2}\right) !} \sqrt{2 j+1} \sqrt{\left(j_{1}+m_{1}\right) !} \\
& \cdot \sqrt{\left(j_{1}-m_{1}\right) !} \sqrt{\left(j_{1}+m_{2}\right) !} \sqrt{\left(j_{1}-m_{2}\right) !} \sqrt{(j+m) !} \sqrt{(j-m) !} \\
& \cdot \sum_{k=p}^{q} \frac{(-1)^{k}}{k !\left(j_{1}+j_{2}-j-k\right) !\left(j_{1}-m_{1}-k\right) !\left(j_{2}+m_{2}-k\right) !\left(j+k+m_{1}-j_{2}\right) !\left(j+k-j_{1}-m_{2}\right) !},
\end{aligned}
$$


where $\delta_{i, j}=1$ if $i=j, \delta_{i, j}=0$ if $i \neq j, p=\max \left(-j+j_{2}-m_{1},-j+j_{1}+m_{2}, 0\right)$ and $q=$ $\min \left(j_{1}-m_{1}, j_{2}+m_{2}\right)$. If $t=3$ then $(7)$ can be reduced to

$$
\begin{aligned}
& P(d, r, b, t)=C(d, b)(-1)^{-\frac{a+5}{3}-\frac{b}{3 d}} \Gamma\left(\frac{a+3}{3}+\frac{b}{3 d}\right) \Gamma\left(\frac{a+4}{3}+\frac{b}{3 d}\right) \sqrt{\Gamma\left(\frac{2-a}{3}\right)} \sqrt{\Gamma\left(\frac{1-a}{3}\right)} \\
& \cdot \sqrt{\Gamma\left(-\frac{a}{3}\right)} \sqrt{\Gamma\left(1-\frac{b}{d}\right)} \sqrt{\Gamma\left(\frac{2}{3}-\frac{b}{3 d}\right)} \sqrt{\Gamma\left(1-\frac{b}{3 d}\right)} \\
& \cdot \sqrt{\Gamma\left(\frac{4}{3}-\frac{b}{3 d}\right)} \sqrt{\Gamma\left(\frac{1-a}{3}+\frac{2 b}{3 d}\right)}\left\{\begin{array}{ccc}
-\frac{b}{3 d} & \frac{b}{6 d}-\frac{a+4}{3} & \frac{a-2}{6}-\frac{b}{6 d} \\
\frac{b}{6 d}-\frac{a+4}{6} & -\frac{1}{3}-\frac{b}{3 d} & -\frac{1}{3}+\frac{b}{3 d}
\end{array}\right\} \\
& /\left[\Gamma\left(\frac{1-a}{3}-\frac{b}{3 d}\right) \sqrt{\Gamma\left(1+\frac{b}{3 d}\right)} \Gamma\left(\frac{1}{3}+\frac{b}{3 d}\right) \sqrt{\Gamma\left(\frac{b}{3 d}\right)}\right. \\
& \left.\cdot \sqrt{\Gamma\left(\frac{a+3}{3}-\frac{2 b}{3 d}\right)} \Gamma\left(\frac{2}{3}+\frac{b}{3 d}\right) \sqrt{\Gamma\left(\frac{a+4}{3}-\frac{2 b}{3 d}\right)}\right],
\end{aligned}
$$

where

$$
C(d, b)=\frac{(a+1) \Gamma\left(1+\frac{b}{d}\right)}{3^{1+\frac{b}{d}}} \frac{\Gamma\left(\frac{a+1}{3}\right)}{\Gamma\left(\frac{a+2+\frac{b}{d}}{3}\right)} \frac{\Gamma\left(\frac{a+2}{3}\right)}{\Gamma\left(\frac{a+3+\frac{b}{d}}{3}\right)} \frac{\Gamma\left(\frac{a+3}{3}\right)}{\Gamma\left(\frac{a+4+\frac{b}{d}}{3}\right)} .
$$

\section{Case $(r+b) /(d t)$ and $(1-r / d) /(t-1)$ are positive integers}

Let $p=(r+b) /(d t)$ and $q=(1-r / d) /(t-1)$. Assume that $p$ and $q$ are positive integers. In this case, (2) can be reduced to the elementary form

$$
\begin{aligned}
P(d, r, b, t)= & C(d, r, b, t) \Gamma\left(1-\frac{b}{d}\right) \sum_{k=1}^{2 t-1} \frac{\Gamma\left(a_{k}+n_{k}\right)}{\left(n_{k}-1\right) ! \Gamma\left(a_{k}-\frac{b}{d}+1\right)} \\
& \cdot \sum_{j=0}^{n_{k}-1} \frac{\left(1-n_{k}\right)_{j}\left(a_{k}\right)_{j}}{j !\left(a_{k}-\frac{b}{d}+1\right)_{j}} \prod_{\ell=1, \ell \neq k}^{2 t-1} \frac{\left(a_{\ell}\right)_{n_{\ell}}\left(a_{k}-a_{\ell}-n_{\ell}+1\right) !}{\left(a_{k}-a_{\ell}\right)_{n_{\ell}}\left(a_{k}-a_{\ell}+1\right)_{j}}
\end{aligned}
$$

where $a_{k}=\frac{a+k}{t}, 1 \leq k \leq t, a_{k}=\frac{a+k+\frac{r}{d}}{t}, t+1 \leq k \leq 2 t-1, n_{k}=p, 1 \leq k \leq t, n_{k}=q$, $t+1 \leq k \leq 2 t-1$ and $C(d, r, b, t)$ is given by $(3)$.

\section{Case $r=t-1, b=1$ and $d=1$}

In this case, (2) can be reduced to the hypergeometric form

$$
P(d, r, b, t)=C(t){ }_{3} F_{2}\left(\frac{1}{t}, \frac{2}{t}, 1 ; \frac{1}{t}+1, \frac{2}{t}+1 ; 1\right),
$$

where

$$
C(t)=\frac{(t-1)^{t+1}}{t !} \prod_{k=0}^{t-2} \frac{\Gamma\left(\frac{k+t}{t-1}\right)}{\Gamma\left(\frac{2+k}{t-1}\right)}
$$


Elementary expressions are possible for particular values of $t$. For example, if $t=2,3,4,5,6,8$ then

$$
\begin{aligned}
P(d, r, b, t) & =2 C(2) \log 2, \\
P(d, r, b, t) & =\frac{2 \pi C(3)}{3 \sqrt{3}}, \\
P(d, r, b, t) & =\frac{C(4)(\pi+2 \log 2)}{4}, \\
P(d, r, b, t) & =-\frac{C(5)}{50} \sqrt{10} \sqrt{5+\sqrt{5}} \pi+\frac{C(5)}{10} \sqrt{2} \sqrt{5+\sqrt{5}}-\frac{2 C(5)}{5} \sqrt{5} \log 2 \\
-\frac{C(5)}{5} & \sqrt{5} \log 5+\frac{2 C(5)}{5} \sqrt{5} \log (5+\sqrt{5}), \\
P(d, r, b, t) & =3^{-\frac{3}{2}} C(6)(\pi+2 \sqrt{3} \log 2), \\
P(d, r, b, t) & =\frac{C(6)}{4} \log 2+\frac{C(6)}{4} \sqrt{2} \log (1+\sqrt{2})+\frac{C(6)}{8} \sqrt{2} \pi,
\end{aligned}
$$

respectively. Also, if $t=7,9,10$ then (8) can be reduced to

$$
\begin{aligned}
& P(d, r, b, t)=-\frac{2 C(7)}{7} \psi\left(\frac{1}{7}\right)+\frac{2 C(7)}{7} \psi\left(\frac{2}{7}\right) \\
& P(d, r, b, t)=-\frac{2 C(9)}{9} \psi\left(\frac{1}{9}\right)+\frac{2 C(9)}{9} \psi\left(\frac{2}{9}\right), \\
& P(d, r, b, t)=-\frac{1}{5} \psi\left(\frac{1}{10}\right)-\frac{C(10)}{5} \gamma-\frac{C(10)}{4} \log 5-\frac{C(10)}{200} \sqrt{10} \sqrt{5+\sqrt{5}} \pi \\
& \quad-\frac{C(10)}{40} \sqrt{2} \sqrt{5+\sqrt{5}} \pi+\frac{C(10)}{10} \sqrt{5} \log 2+\frac{C(10)}{20} \sqrt{5} \log 5 \\
& \quad-\frac{C(10)}{10} \sqrt{5} \log (5+\sqrt{5}),
\end{aligned}
$$

respectively, where $\gamma$ is Euler's constant and $\psi(x)=d \log \Gamma(x) / d x$ denotes the digamma function.

\section{Acknowledgments}

The author would like to thank the Editor and the referee for careful reading and comments which greatly improved the paper.

\section{References}

[1] Antal, T., Ben-Naim, E. and Krapivsky, P. (2010). First passage properties of the Pólya urn process. Journal of Statistical Mechanics: Theory and Experiment, 7, P07009

[2] Eggenberger, F. and Pólya, G. (1923). Über die Statistik verketteter Vorgange. ZAMM, 3, $279-289$.

[3] Gradshteyn, I. S. and Ryzhik, I. M. (2014). Tables of Integrals, Series and Products, eighth edition. Academic Press, New York.

[4] Mahmoud, H. M. (2009). Pólya Urn Models. CRC Press, Boca Raton, Florida. 
[5] Prudnikov, A. P., Brychkov, Y. A. and Marichev, O. I. (1986). Integrals and Series, volumes 1, 2 and 3. Gordon and Breach Science Publishers, Amsterdam.

[6] Schulte-Geers, E. and Stadje, W. (2015). Maximal percentages in Pólya's urn. Journal of Applied Probability, 52, 180-190. 